\title{
An in vitro model of Mycobacterium leprae induced granuloma formation
}

\author{
Hongsheng Wang ${ }^{1,2}$, Yumi Maeda $^{2 *}$, Yasuo Fukutomi ${ }^{2}$ and Masahiko Makino ${ }^{2}$
}

\begin{abstract}
Background: Leprosy is a contagious and chronic systemic granulomatous disease caused by Mycobacterium leprae. In the pathogenesis of leprosy, granulomas play a key role, however, the mechanisms of the formation and maintenance of $M$. leprae granulomas are still not clearly understood.

Methods: To better understand the molecular physiology of $M$. leprae granulomas and the interaction between the bacilli and human host cells, we developed an in vitro model of human granulomas, which mimicked the in vivo granulomas of leprosy. Macrophages were differentiated from human monocytes, and infected with $M$. leprae, and then cultured with autologous human peripheral blood mononuclear cells (PBMCs).

Results: Robust granuloma-like aggregates were obtained only when the M. leprae infected macrophages were co-cultured with PBMCs. Histological examination showed M. leprae within the cytoplasmic center of the multinucleated giant cells, and these bacilli were metabolically active. Macrophages of both $\mathrm{M} 1$ and $\mathrm{M} 2$ types co-existed in the granuloma like aggregates. There was a strong relationship between the formation of granulomas and changes in the expression levels of cell surface antigens on macrophages, cytokine production and the macrophage polarization. The viability of $M$. leprae isolated from granulomas indicated that the formation of host cell aggregates benefited the host, but the bacilli also remained metabolically active.
\end{abstract}

Conclusions: A simple in vitro model of human M. leprae granulomas was established using human monocyte-derived macrophages and PBMCs. This system may be useful to unravel the mechanisms of disease progression, and subsequently develop methods to control leprosy.

Keywords: Mycobacteria, Leprosy, Granuloma

\section{Background}

Leprosy is a chronic mycobacterial infection that presents an extraordinary range of cellular immune responses in humans. Regulation of cell-mediated immunity against Mycobacterium leprae through the fine-tuning between cells, cytokines and chemokines continues to be unraveled. Similar to other mycobacterial infections, granulomatous inflammation in the skin lesion defines certain forms of leprosy $[1,2]$. The bacilli enter and replicate within macrophages, resulting in the production of cytokines and chemokines, which in turn triggers an inflammatory response leading to the recruitment of macrophages and lymphocytes at the infectious site. Granulomas mainly contain

\footnotetext{
*Correspondence: yumi@nih.go.jp

${ }^{2}$ Department of Mycobacteriology, Leprosy Research Center, National Institute of Infectious Diseases, 4-2-1 Aobacho, Higashimurayama, Tokyo 189-0002, Japan

Full list of author information is available at the end of the article
}

macrophages, epithelioid cells (ECs), multinucleated giant cells (MGCs), surrounded by a rim of $\mathrm{T}$ lymphocytes [3]. The organization and the cellular constituents of the developing $M$. leprae granulomas vary with the status of the host immune response. Presumptively, granulomatous lesions can be categorized within two polar forms [4]. At one extreme, tuberculoid granulomas are organized as nodular lesions with ECs and MGCs in the lesion center surrounded by a rim of fibrous connective tissue, lymphocytes along the periphery of the granuloma, and acid-fast bacilli are rarely demonstrable in the lesions. At the other extreme, the pathological feature of lepromatous leprosy skin lesions are characterized by a lack of organization of cells, with very high numbers of foamy macrophages containing very large numbers of bacilli, and disorganized lymphocyte infiltration.

\section{Biomed Central}

(c) 2013 Wang et al.; licensee BioMed Central Ltd. This is an Open Access article distributed under the terms of the Creative Commons Attribution License (http://creativecommons.org/licenses/by/2.0), which permits unrestricted use, distribution, and reproduction in any medium, provided the original work is properly cited. 
Granulomas have long been believed to benefit the host by containing and restricting the growth of mycobacteria in a localized area, to prevent the spread of the disease to other parts of the tissue or organs [5]. However, some studies in zebra fish infected with $M$. marinum and $M$. tuberculosis suggested that the granulomas contribute to early bacterial growth and expanding infection [6-10].

The structure, function, and evolution of granulomas have been studied using various animal models [11,12], high-resolution chest computed tomography scans of pulmonary tuberculosis patients [13], and explanted tissues $[5,14]$. Interestingly, the in vitro models of human mycobacterial granulomas have been studied by infection with Bacillus Calmette-Guérin (BCG) or stimulation with antigens such as purified protein derivatives or artificial beads coated with mycobacterial components $[15,16]$. These studies have identified infected macrophages, ECs, and several types of MGCs, which are thought to play important roles in the formation and maintenance of granulomas. In addition, macrophages demonstrate considerable plasticity that allows them to efficiently respond to environmental signals. These cells are generally classified as M1 (classic) macrophages, which produce proinflammatory cytokines and mediate resistance to pathogens and contribute to tissue destruction, or M2 (alternative) macrophages, that produce anti-inflammatory cytokines and promote tissue repair [17-19]. However, so far, we know little about the relationship between the polarization of macrophages within mycobacterial granulomas.

In this study, we developed an in vitro model of $M$. leprae granulomas, which mimicked the human granulomatous skin lesion with progressive recruitment of monocytes around macrophages infected by $M$. leprae, and their differentiation into ECs and MGCs as well as recruitment of activated lymphocytes. This model may be useful for unravelling the mechanisms of disease progression, and find effective strategies to control the spread of bacilli.

\section{Methods}

\section{Ethics statement, cell culture and preparation of the} bacteria

Peripheral blood was obtained from healthy Japanese individuals with informed consent. The study was approved by the ethics committee of the National Institute of Infectious Diseases (NIID). In Japan, BCG vaccination is compulsory for children aged 0-4 years old. Macrophages were differentiated from monocytes using granulocyte-macrophage colony-stimulating factor (GM-CSF) as described previously [20,21]. Animal experiments were carried out in strict accordance with the recommendations of Japan's Animal Protection Law. The protocol was approved by the Experimental Animal Committee of NIID Tokyo (Permit
Number: 211002). M. leprae (Thai-53 strain) was propagated in athymic BALB/c-nu/nu mice (Clea Co, Tokyo) [22]. At 8-9 months post-infection, mouse footpads were processed to recover $M$. leprae [23]. For all experiments, $M$. leprae was freshly prepared. Human cells without the bacilli were cultured at $37^{\circ} \mathrm{C}$ but when infected with the bacilli, the cells were cultured at $35^{\circ} \mathrm{C}$ to maintain the viability of $M$. leprae in host cells.

\section{Culture of macrophages and peripheral blood mononuclear cells for the formation of cellular aggregates}

Macrophages, differentiated from monocytes using GMCSF after 4 days culture in RPMI containing 20\% fetal calf serum (FCS) were transferred into 24-well tissue culture plates (Falcon) $\left(1 \sim 2 \times 10^{5}\right.$ cells/well). Freshly prepared $M$. leprae were then added to each well. The multiplicity of infection (MOI: 50) was determined based on the assumption that macrophage were equally susceptible to infection with $M$. leprae [24]. After $24 \mathrm{hr}$, autologous peripheral blood mononuclear cells (PBMCs) were cultured with $M$. leprae infected macrophages at a ratio of 5:1 (PBMCs: macrophages). In some cases, macrophages were infected with $M$. leprae without PBMCs and in others, macrophages and PBMCs were co-cultured and macrophages alone were used as negative controls. The cells were cultured at $35^{\circ} \mathrm{C}$ for periods from $24 \mathrm{~h}$ to 10 days with medium changes every other day. To detach the cells from plates TrypLE Express (Gibco) was used, and then the cells were maintained in medium containing $10 \%$ FCS for $30 \mathrm{~min}$, before processing for flow cytometric analyses. In other experiments we have also isolated $\mathrm{T}$ lymphocytes and monocytes were isolated using Dynabeads Untouched Human T cells and Dynabeads MyPure Monocyte kit 2 (Invitrogen), and used instead of PBMCs.

\section{Phase-contrast microscopy and fluorescence microscopy}

Macrophages grown on a 13-mm coverslip in a 24-well plate, were infected with $M$. leprae for $24 \mathrm{~h}$. Autologous PBMCs were then co-cultured with macrophages for additional 9 days. Macrophages were fixed in 2\% paraformaldehyde, or methanol pre-chilled to $-20^{\circ} \mathrm{C}$, and then observed under a phase-contrast microscope (Olympus CKX41 with $\times 10$ and $\times 20$ objective lenses). Photographs were taken with an Olympus DP50 system. Image acquisition and data processing were performed using DP controller software. In other experiments, cells were stained with May-Grünwald-Giemsa stain (MGG) (Sigma-Aldrich) or by $\mathrm{TB}$ Carbolfuchsin $\mathrm{ZN}$ stain according to the manufacturer's instructions (BD Biosciences).

Cell imaging was performed using LSM5-Exciter laser scanning microscope equipped with a $568 \mathrm{~nm}$ laser (Carl Zeiss). Fixed cells were stained with anti-human CD163 monoclonal antibody (mAb: BioLegend) and the 
secondary antibody used was an Alexa Fluor 568conjugated goat anti-mouse IgG (Invitrogen/Molecular Probes). Nuclei were counterstained with Hoechst 33342 dye (Sigma-Aldrich). M. leprae was stained by auramine $\mathrm{O}$ (BD Biosciences). Images were obtained under a fluorescence confocal microscope. Data were processed using LSM software ZEN 2007.

\section{Analysis of cell surface antigens on macrophages by flow cytometry and microscopy}

Macrophages were collected after time points of 1 and 9 days of co-culture with the PBMCs or M. leprae stimulation. The expression of cell surface antigens on macrophages, was analyzed using a FACSCalibur flow cytometer (BD Biosciences). Dead cells were eliminated from the analysis by staining with 7 -amino actinomycin D. For the analysis of cell surface antigens, the following mAb were used: FITC-conjugated mAb against CD68 (KP) was purchased from Dako, FITC conjugated TLR4 (HTA125) and CD206 (19.2), and PE conjugated mAb against CD86 (FUN-1) was all purchased from BD Biosciences and PE conjugated mAb to CD14 (HCD14) and CD163 (RM3/1) were from BioLegend. The numbers in the insets indicate the mean fluorescent values of the cells stained with the respective mAbs.

\section{Determination of cytokine levels}

The levels of the cytokines: Interferon (IFN)- $\gamma$, interleukin (IL)-2, tumor necrosis factor (TNF)- $\alpha$, IL-12p40, IL-1 $\beta$ and IL-10 in the culture supernatants were quantified using enzyme assay kits, OptEIA Human ELISA Set (BD Biosciences) and processed according to the manufacturer's instructions. IL-4 and IL-13 was purchased from MABTECH AB. Cytokine levels were expressed as $\mathrm{pg}$ of protein/ml of protein. Real-time PCR analysis of mRNA extracted using an RNeasy Mini kit (Qiagen), was performed using SYBR Green PCR Master Mix (Applied Biosystems) with specific primers according to the manufacturer's instructions. The instrument used for the detection of the expression of mRNA was StepOnePlus with StepOne software.

\section{Determination of $M$. leprae viability}

The viability of $M$. leprae recovered from the macrophages of different groups was detected by radiorespirometry, that measures the oxidation of ${ }^{14} \mathrm{C}$ palmitic acid to ${ }^{14} \mathrm{CO}_{2}$, as described previously [25]. Briefly, the adherent macrophages and granulomas with bacilli were lysed in $300 \mu \mathrm{l}$ of a $0.1 \mathrm{~N} \mathrm{NaOH}$ solution to release intracellular $M$. leprae. After neutralization with $0.1 \mathrm{~N} \mathrm{HCl}$ solution, an equal volume of 2 times concentrated Middlebrook 7H9 broth was added. ${ }^{14} \mathrm{C}$ labeled palmitic acid was added to the lysates of macrophages or granulomas, followed by incubation at $33^{\circ} \mathrm{C}$. After 7 days, cumulative amounts of oxidized palmitic acid released as ${ }^{14} \mathrm{CO}_{2}$ by metabolically active $M$. leprae were measured using a Packard 1500 TRI-CARB liquid scintillation analyzer. The unpaired Student's t-test was used to determine the statistical significance of the two data sets.

\section{Results \\ Granuloma-like aggregates formed by co-culture of $M$. leprae infected macrophages and autologous PBMCs}

When PBMCs were incubated with $M$. leprae infected macrophages in a 24-well tissue culture plate, the cells aggregated to form a multilayered granuloma-like aggregates by day 9 as shown in Figure 1A, whereas control groups did not recruit any cells at this stage (Figure 1B, C). We observed formation of a granular ball-like structure caused by some synapses around aggregates. These in vitro granulomas exhibited a cellular structure similar to that in histopathological specimens of tuberculoid leprosy lesions showing $\mathrm{T}$ lymphocytes surrounding the differentiated, ECs and MGCs that may be involved in cytokine production for intercellular communication. (Figure 1D). Confocal microscopic analysis of $M$. lepraeinduced granuloma showed a multilayered structure (about 3-4 cell layers in transverse and straight sections), and some cells were positive for CD163 (red), a macrophage marker (Figure 1E).

\section{Characterization of the cell populations recruited within in vitro granuloma-like aggregates}

To identify and characterize the different cell types in granuloma-like aggregates, the cells were plated on glass slides and stained on day 9 of co-culture. MGG staining showed that activated macrophages with larger cytoplasm, and MGCs were observed, which resembled those in the granulomas of leprosy (Figure 2B, D). MGCs are thought to be formed as a result of fusion of macrophages, monocytes and ECs (Figures 2A, C). The presence of $M$. leprae in MGCs was confirmed by staining with TB Carbolfuchsin ZN (arrows in Figure 2E, F). In addition, confocal microscopy revealed the presence of MGCs with auramine $\mathrm{O}$ stained $M$. leprae, in the cytoplasmic region (Figure 2G, H). To characterize macrophages, ECs and MGCs in the granuloma-like aggregates, we performed immunofluorescence staining for macrophage markers CD68, CD1a and CD163 (data not shown). Both the macrophages and the MGCs could express the CD68 and CD1a marker, but the expression level of CD68 on the macrophages was higher than that on the MGCs. With the increasing number of nuclei in MGCs, lower levels of CD68 was observed (not shown), although there was no significant difference in the expression levels of CD1a between macrophages and MGCs. These data indicate that MGCs belong to the monocyte/macrophage lineage. 

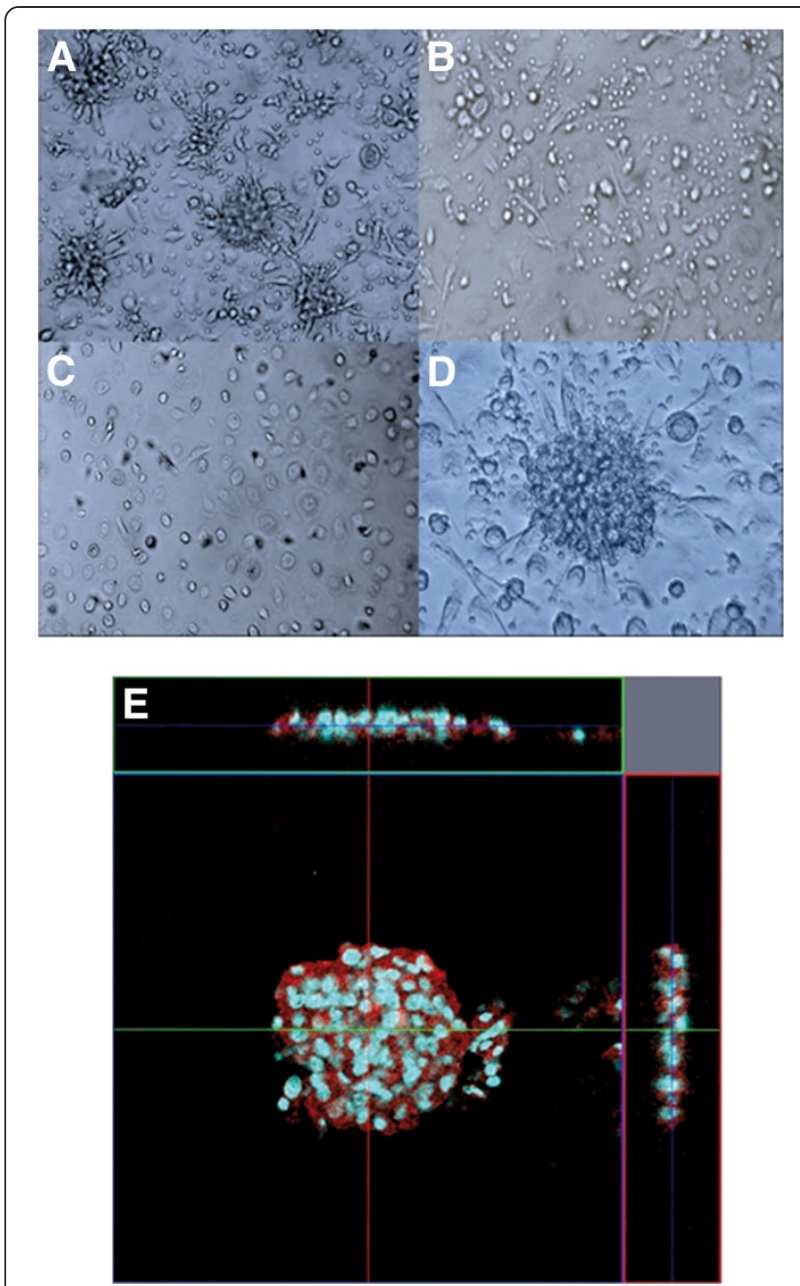

Figure 1 Formation of granuloma-like cellular aggregates by co-culture of PBMCs and macrophages infected with $M$. leprae. (A) Co-culture of macrophages $\left(1 \times 10^{5}\right)$, infected with $M$. leprae (MOl:50) and autologous PBMCs $\left(5 \times 10^{5}\right)$ in a 24 well-plate resulted in the formation of granuloma-like aggregates by day 9. (B) Culture of macrophages $\left(1 \times 10^{5}\right)$ and autologous PBMCs $\left(5 \times 10^{5}\right)$ for 9 days, without the bacilli. No formation of granuloma-like aggregates was observed. (C) Macrophages $\left(1 \times 10^{5}\right)$ infected with M. leprae (MOI:50) after 9 days co-culture. (D) Higher magnification (2X) of the cell-aggregates in (A). (E) Confocal microscopic (LSM5 Exciter) analysis of $M$. leprae-induced granuloma revealed a multilayered structure (about 3-4 cell layers cells in transverse and straight sections). The cells in aggregates were positive for CD163 (red), a marker of macrophages. Nuclei were stained with Hoechst 33343 (blue). Representative data from a single donor are shown.

Expression levels of cell surface antigens on macrophages at different time points

We investigated the expression levels of cell surface antigens on macrophages from different groups at two different time points, day 1 and day 9. On day 1, there was no significant difference in the expression of cell surface antigens on macrophages between groups. Compared with day 1 macrophages, day 9 macrophages, which were infected with $M$. leprae and co-cultured with PBMCs to

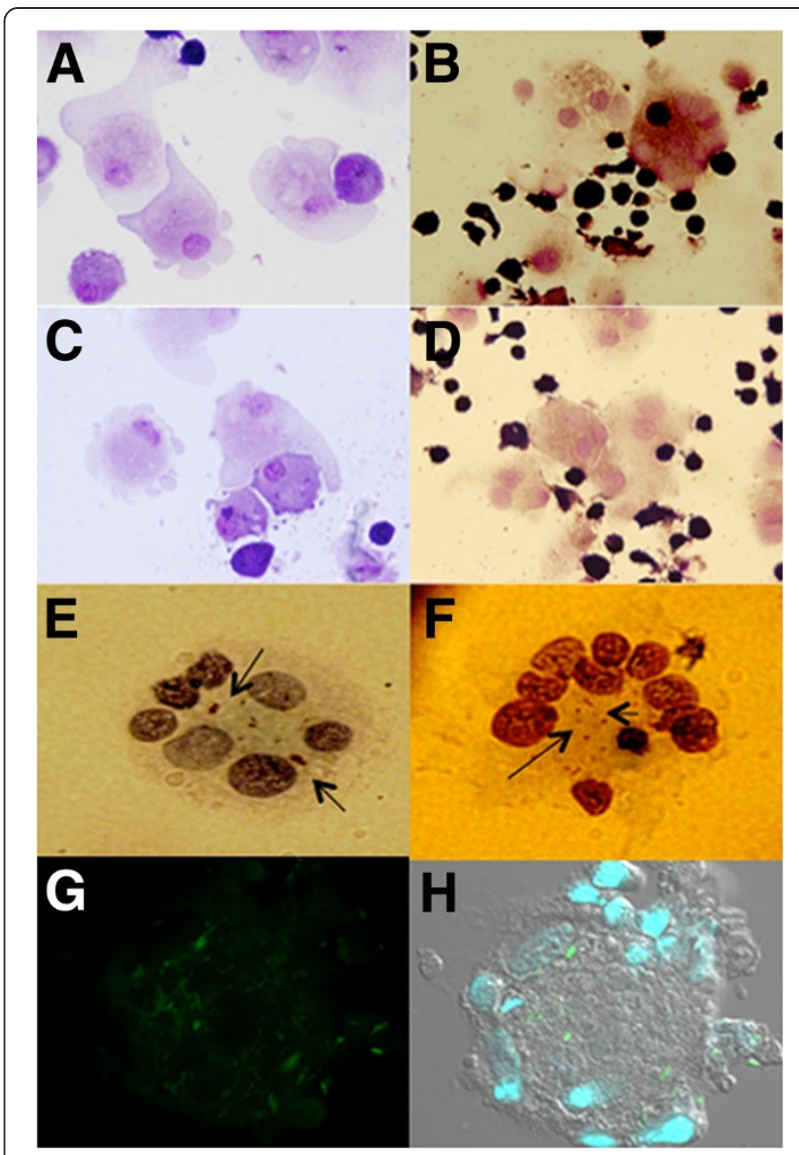

Figure 2 Cell populations in granuloma-like aggregates.

May-Grünwald-Giemsa (MGG) staining showed that there are mainly macrophages, ECs $(\mathbf{A}, \mathbf{D})$ and MGCs in the aggregates $(\mathbf{B}, \mathbf{D})$ MGCs were formed by the intercellular fusion and phagocytosis of cells $(\mathbf{C})$. M. leprae were stained with Ziehl-Neelsen (shown with arrows) and the bacilli were found to be restricted to the central cytoplasmic region of the MGCs (E, F). Confocal microscopy of MGCs showed $M$. leprae stained with auramine $O$ (green) and the nuclei stained with Hoescht $(\mathbf{G}, \mathbf{H})$.

form granuloma-like aggregates, showed higher expression of CD14 (pattern recognition receptor), CD68 (macrophage marker related to phagocytic activities), CD163 (scavenger receptor) and CD206 (mannose receptor), although the expression of major histocompatibility complex (MHC) class-II, CD86, and toll-like receptor (TLR)-4 did not change (Figure 3). Interestingly, in our long-term culture (9 days) of macrophages infected with $M$. leprae, the expression of CD14, CD68, CD163, TLR4, CD86 and CD206 was significantly lower than that in macrophages infected with $M$. leprae and co-cultured with PBMCs. CD206 expression was the lowest in macrophages cocultured with PBMCs, although CD163 expression was significantly high (Figure 3). CD163 and CD206 are markers of M2 macrophages, whereas CD86 expression is associated with M1 macrophages. Therefore, the M1 and M2 macrophages appeared to coexist in granulomas. 


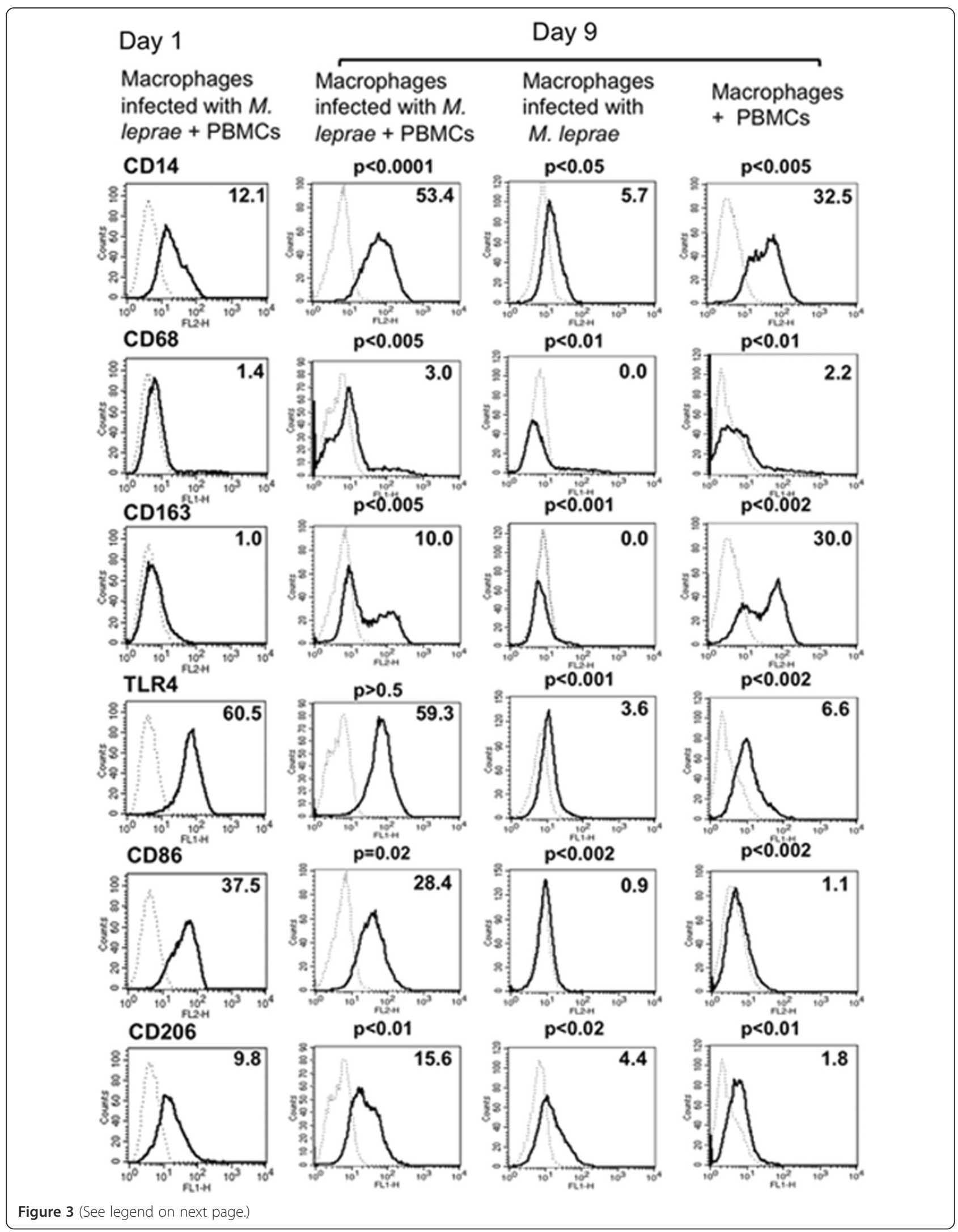


(See figure on previous page.)

Figure 3 Expression of cell surface antigens on macrophages at two different time points. Compared with the control group on day 1 , day 9 macrophages infected with M. leprae and co-cultured with T lymphocytes showed relatively higher expression of CD14, CD163 and CD206. While in macrophages infected with M. leprae, the expression level of CD14, CD68, CD163, TLR4, CD86 and CD206, were downregulated as compared to those infected macrophages co-cultured with PBMCs. Representative data of one donor, from three independent experiments are shown. P-values were calculated using the Welch unpaired t-test in comparison with day 1 macrophages.

\section{Cytokines in culture supernatants}

The culture supernatants from different groups were collected on days 1,3 and 9 after the start of macrophage culture. The release of IFN- $\gamma$, IL- 2, TNF- $\alpha$, IL12p40, IL-1 $\beta$, IL-4 and IL-13, was evaluated by ELISA (Figure 4). Interestingly, the expression levels of the various cytokines in supernatants, from different groups showed significant differences that were associated with the formation of granuloma-like aggregates and changes of cell surface antigen expression on macrophages. In the group with $M$. leprae infected macrophages cocultured with PBMCs, the concentrations of IL-2, IL-1 $\beta$ and TNF- $\alpha$ peaked on day 1 after infection and then declined gradually. The level of IL-12 p40 also declined slowly by day 9 . IFN- $\gamma$ levels were low on day 1 , but increased 7 fold by day 4, and then remained unchanged till day 9. A high level of IL-10 expression in macrophages and macrophages cultured with PBMCs was observed, but the expression was significantly decreased when macrophages were infected with $M$. leprae as observed in the day 9 cytokine expression levels. However, when macrophages were differentiated with M-CSF, the expression of IL-10 was significantly high when macrophages were infected with $M$. leprae (Additional file 1: Figure S1). IL-4 and IL-13 were not detected in any groups on days 1 and 9 from the start of macrophage culture (data not shown). Real time PCR results further confirmed the cytokine expression and showed similar results except for the IL- 2 and TNF- $\alpha$, whose expression was observed in control groups of macrophages infected with $M$. leprae in addition to those co-cultured with PBMCs (Figure 5).

The viability of $M$. leprae in granuloma-like aggregates We determined the viability of $M$. leprae at days 1 and 9, when granuloma-like aggregates were observed in cocultures of $M$. leprae infected macrophages with PBMCs, whereas in cultures of macrophages infected with $M$. leprae, there was no granuloma formation. The amount of radioactive $\mathrm{CO}_{2}$ evolved which reflects the rate of ${ }^{14} \mathrm{C}$-palmitic acid oxidized by $M$. leprae, which was measured by a scintillation counter. No significant difference in ${ }^{14} \mathrm{CO}_{2}$

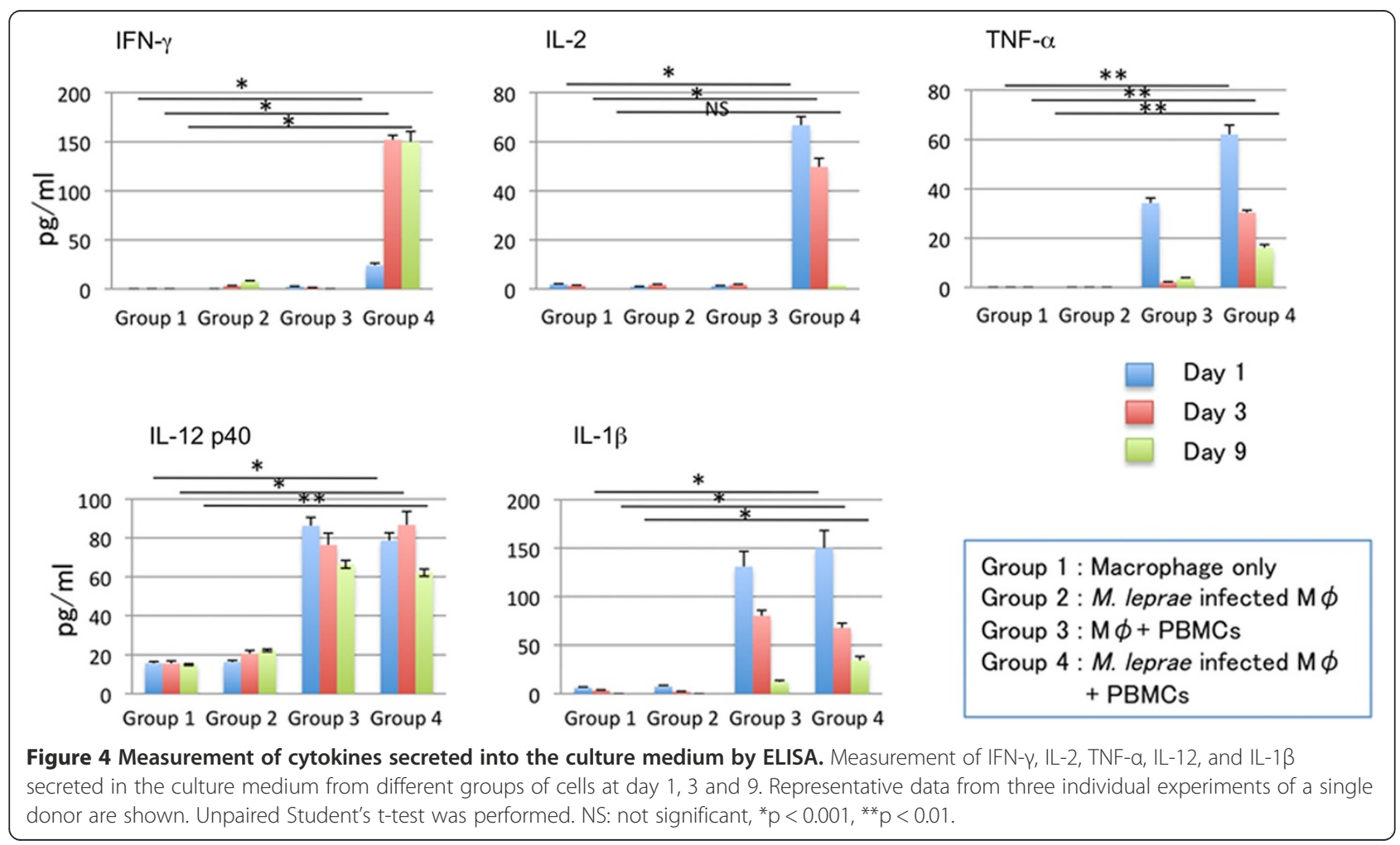




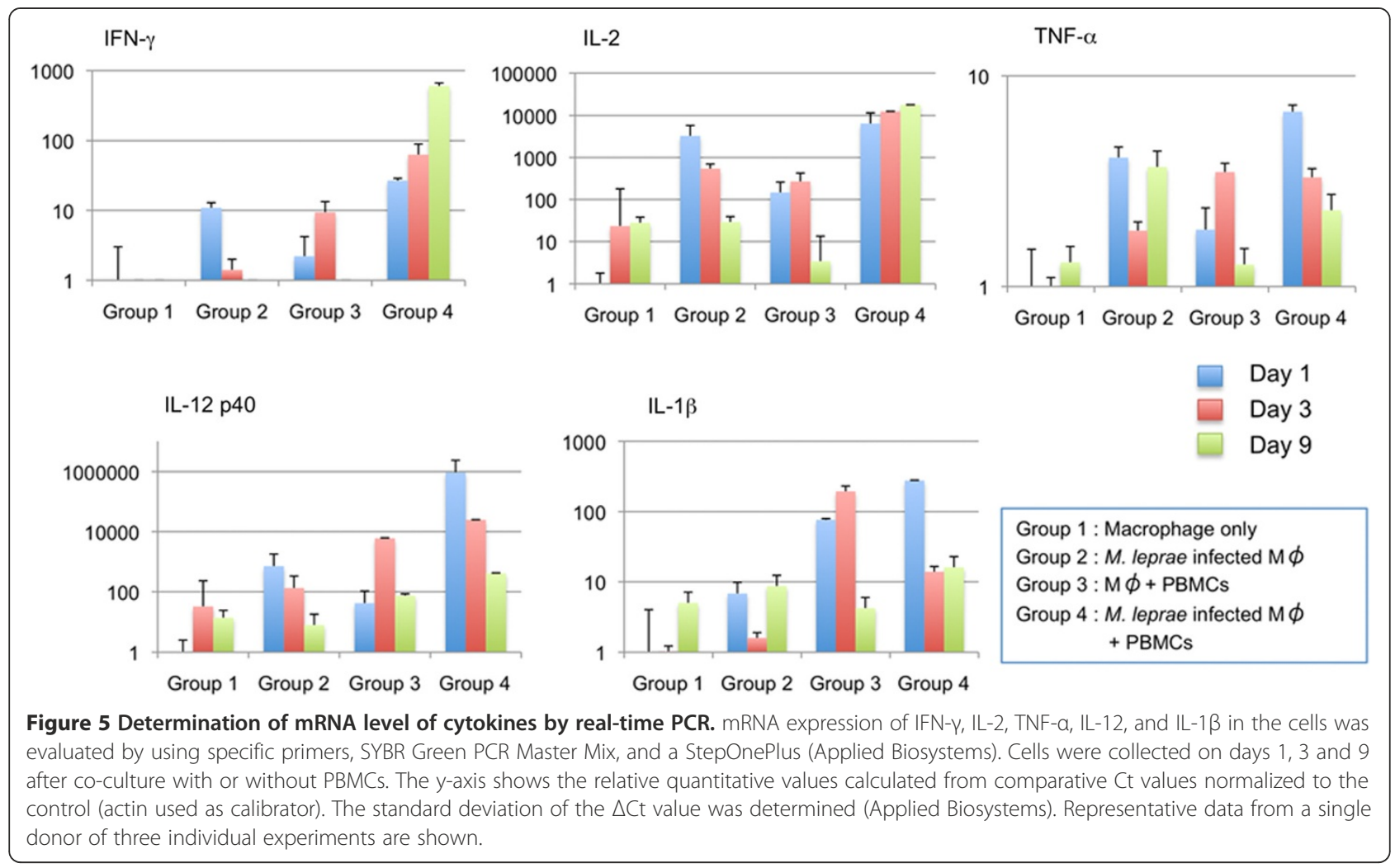

production was observed from macrophage in either groups on days 1, and 9. However, the amount of radioactive $\mathrm{CO}_{2}$ released from macrophages infected with $M$. leprae and cocultured with PBMCs for 9 days was lower but not significantly lower than that released from macrophages infected with $M$. leprae alone (Figure 6).

\section{Discussion}

In the 1960s, Ridley and Jopling proposed a histological classification scheme for leprosy [26]. At one extreme, called the polar tuberculoid, leprosy patients show a high degree of cell-mediated immunity, lesions revealing welldeveloped granulomatous inflammation and rarely acidfast bacilli are detected. At the other extreme, termed polar lepromatous patients have no apparent resistance to M. leprae, and skin biopsies reveal sheets of foamy macrophages in the dermis containing very large numbers of bacilli and microcolonies called globi. Currently, the formation and maintenance of granulomas are considered to be critical components of the host response to M. leprae infection, which determine not only whether primary disease occurs, but also the clinical manifestation. Granuloma formation is studied in mouse models but little is known about the human granuloma due to the ethical problems of using human samples and the difficulties in establishing a good model using human cell lines.

The formation of small, rounded granuloma-like-structure, was previously described by co-culture of blood lymphocytes with autologous macrophages infected with M. tuberculosis, or BCG or stimulation with other mycobacterial antigen such as purified protein derivatives. These granuloma-like structures showed abundance of CD68 positive macrophages with small round lymphocytes scattered throughout the

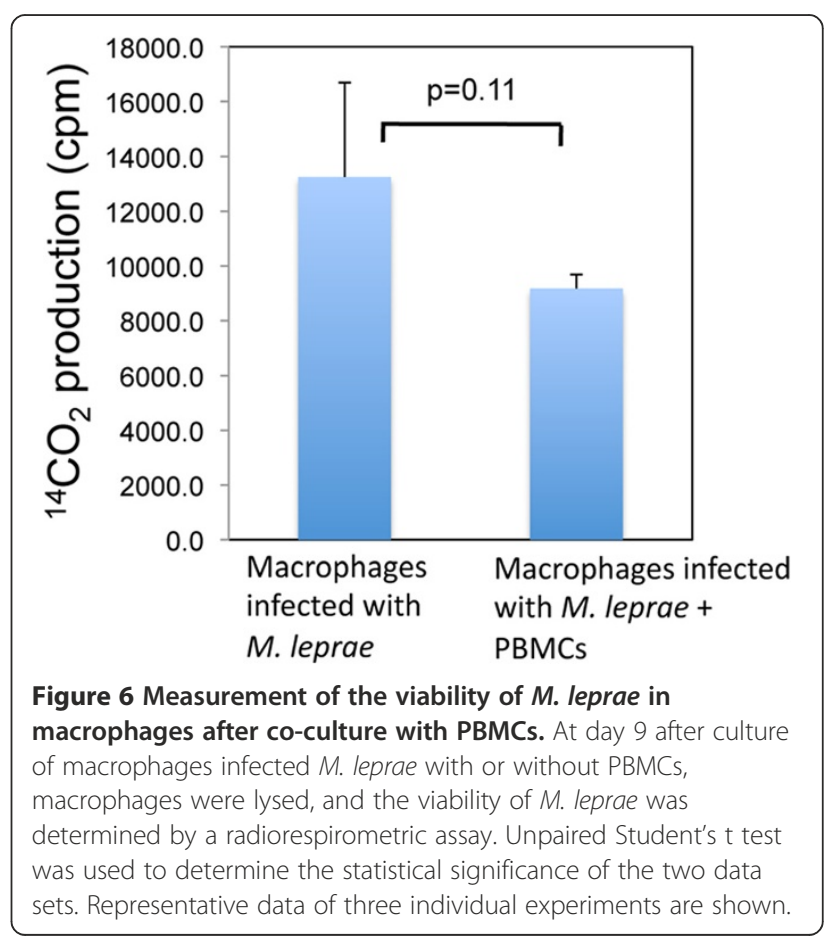


granuloma $[15,16]$. These models not only exhibit structural similarities to granulomas observed in human clinical specimens, but also show patterns of cell antigen expression and/ or cytokine production that appear consistent with those observed in tuberculosis patients. However, the formation of granulomas in leprosy, involving $M$. leprae infection has not been previously studied in vitro. The only data available on granuloma formation of leprosy is from the immunological staining of biopsies of patients, and granulomas harvested from the footpads of athymic nude mice [27].

In our model, we first infected the immature human macrophages with $M$. leprae. To mimic the recruitment of additional PBMCs which would occur in vivo, autologous PBMCs were added after $24 \mathrm{~h}$ and cultured at $35^{\circ}$ $\mathrm{C}$, the optimal temperature for the growth of $M$. leprae and macrophages to be kept viable. Within 9 days of culture, macrophages and $\mathrm{T}$ lymphocytes gathered to form a granuloma-like aggregates with fused macrophages, appearing as multinucleated cells, and epitheloid macrophages tightly linked to surrounding macrophages and lymphocytes. However, in control groups, the formation of granuloma-like aggregates was not observed. When autologous $\mathrm{T}$ lymphocytes and monocytes were purified and used instead of PBMCs, a similar formation of granuloma like aggregates were observed, together with production of the same amounts of cytokines, indicating that $\mathrm{T}$ lymphocytes and monocytes are sufficient for the containment of $M$. leprae in granuloma like structures.

Electron microscopy studies indicated that the tuberculoid lesion had an appearance of a granulomatous response with a predominance of ECs and MGCs, and the mononuclear phagocytes which are surrounded by a mantle of lymphocytes [28]. In the present in vitro model of granulomas, MGCs were prominent, and resembled MGCs observed in a tuberculoid lesion. MGCs have been described by Langhans, but the function of these cells in the granuloma remains to be elucidated [29]. In this study, we observed not only Langhans giant cells (MGCs with a circular nuclear organization in contrast to the MGCs formed in response to a foreign body that lacks this kind of organization), but also the bacilli surrounded by nuclei and restricted to the central cytoplasmic region. Because this type of MGC is not observed in the normal mouse model, it is interesting to further focus on the formation, mechanism and function of such MGCs using human in vitro model or humanized mouse model as recently described by Heuts et al. [30]. The in vitro model of leprosy granulomas still needs to be investigated, and compared to that obtained using leprosy patients' monocytes and T cells.

Macrophages function as control switches of the immune system, providing a balance between pro- and anti-inflammatory responses by developing into subsets of M1 or M2 activated macrophages. M1 macrophages are activated by type I cytokines such as IFN- $\gamma$ and TNF $\alpha$, Alternatively, activated M2 macrophages are subdivided further into M2a (activated by IL-4 or IL-13), M2b (activated by immune complexes in combination with IL-1 3 ) and M2c (activated by IL-10 or glucocorticoids) [31]. M1 macrophages exhibit a potent microbicidal activity, and release IL-12, promoting strong Th1 immune responses. It is the M1 population that is thought to contribute to macrophage-mediated tissue injury [19,32]. In contrast, M2 macrophages support Th2-associated effector functions and exert a selective immunosuppressive activity. M2 macrophages also play a role in the resolution of inflammation through phagocytosis of apoptotic neutrophils, reduced production of pro-inflammatory cytokines, and increased synthesis of mediators that are important for tissue remodeling and wound repair. We investigated the contribution of the macrophage polarization, MGC formation and immune responses against $M$. leprae in granulomas, and found that there was a strong relationship between the formation of granuloma-like aggregates, the changes of cell surface antigen expression on macrophages, and the expression levels of various cytokines with the macrophage polarization. In $M$. leprae infected macrophages cocultured with PBMCs, the concentrations of IL-2, IL-12 and TNF- $\alpha$ peaked at day 1 , while, TLR4, CD86, and $\mathrm{MHC}$ molecules were highly expressed, indicating that most of the macrophages were of the M1 subset. At day 9, in the same group of infected macrophages cocultured with PBMCs, the cells assembled and formed a multilayer, granuloma-like aggregates, and the macrophages not only highly expressed TLR4 and CD86, but also scavenger receptor (CD163) and mannose receptor (CD206) molecules. CD163 and CD206 are the markers of M2 macrophages. Therefore, the M1 and M2 macrophages coexisted in granuloma-like aggregates. Consistent with this observation, the levels of IL-1 $\beta$, IL-2, IL-12 and IFN $-\gamma$ were high in the culture medium, promoting the differentiation of macrophages into both M1 and M2 subsets. The protective cell mediated immune response is regulated by the cytokine equilibrium, while the tuberculoid pole is characterized by the presence of Th1 cytokines (IL-2, IFN- $\gamma$, TNF- $\alpha$ and IL-12), and lepromatous is characterized by type 2 cytokines (IL-4, IL-6 and IL-10) [33]. Because IL-10 is an immunosuppressive cytokine implicated in susceptibility to mycobacterial infection, we examined the expression of IL-10 in more detail. Indeed, the infection with $M$. leprae suppressed the production of IL-10. However, when macrophages were differentiated with M-CSF, rather than GM-CSF, $M$. leprae infection further enhanced IL-10 production. Our results indicate that the granuloma aggregates studied here, are similar to those observed in the tuberculoid form of leprosy. However, little is known about the type 
of cytokines that influence the formation of macrophages for containment of $M$. leprae in the granulomas during the pathogenesis of leprosy.

We also investigated the viability of $M$. leprae in macrophages at different time points. At day 9, a number of granuloma-like aggregates were observed in co-cultures of PBMCs with macrophages infected with M. leprae. However in macrophages infected with $M$. leprae without the PBMCs, granuloma-like aggregates were not observed. There were no significant differences in the viability of $M$. leprae in macrophage of different groups on day 1 , but on day 9 , the viability of $M$. leprae in the group that formed granuloma-like aggregates was slightly lower, although not significantly, than that of $M$. leprae in infected macrophages without PBMCs. Evidently, granuloma-like aggregates appear to benefit the host but the bacilli remained metabolically active. The mechanism of this phenomenon needs further in-depth analysis.

\section{Conclusions}

In summary, we have developed for the first time a method to obtain in vitro $M$. leprae granulomas using human monocyte derived macrophages and PBMCs. Using this model, we obtained some basic information about the characteristics of in vitro granulomas. In addition, the viability of $M$. leprae in granuloma-like aggregates remained unaltered during the culture period. Effective strategies to allow the bacilli to succumb to the formation of granuloma may assist in the primary control of the infection.

\section{Additional file}

Additional file 1: Figure S1. Measurement of IL-10 secreted into the culture medium by ELISA. Measurement of IL-10 secreted in the culture medium from different groups of cells at days 2, 6 and 9 is shown. Two types of macrophages were used to analyze the data. (A) Macrophages differentiated using GM-CSF, and (B) macrophages differentiated from monocytes using M-CSF. Representative data from two individual experiments of a single donor are shown. Unpaired student's $t$ test was performed, ${ }^{*} p<0.0001,{ }^{* *} p<0.001,{ }^{* * *} p<0.05$.

\section{Abbreviations}

DCs: Dendritic cells; PBMCs: Peripheral blood mononuclear cells; ECs: Epitheloid cells; MGCs: Multinucleated giant cells; BCG: Bacillus Calmette- Guérin.

\section{Competing interests}

The authors declare that they have no competing interests.

\section{Authors' contributions}

HW, YM participated in the design of the study and carried out the cell culture experiments, YF carried out the confocal microscopic examination, and radio-respirometric assay. HW, YM, and MM were involved in the preparation of the manuscript. All authors have read and approved the final manuscript.

\section{Acknowledgments}

This study was supported by grants from the Grant-in-Aid from the Ministry of Health, Labor and Welfare of Japan for "Research on Emerging and Re-emerging Infectious Diseases" (Grant no. H24-Shinko-Ippan-009 to YM) and also from the National Natural Science Foundation of China (30972651), the fund for Key Clinical Program of the Ministry of Health (2010-2012-125). We appreciate the helpful assistance of Drs. Masanori Matsuoka and Masanori Kai for the M. leprae propagation and isolation. We also thank the Japanese Red Cross Society for kindly providing whole blood cells from healthy donors.

\section{Author details}

'Institute of Dermatology, Chinese Academy of Medical Sciences and Peking Union Medical College, 12 Jiangwangmiao Road, Nanjing 210042, China. ${ }^{2}$ Department of Mycobacteriology, Leprosy Research Center, National Institute of Infectious Diseases, 4-2-1 Aobacho, Higashimurayama, Tokyo 189-0002, Japan.

Received: 13 December 2012 Accepted: 17 June 2013 Published: 20 June 2013

\section{References}

1. Britton WJ: Immunology of leprosy. Trans R Soc Trop Med Hyg 1993, 87:508-514.

2. Kaplan G, Cohn ZA: The immunobiology of leprosy. Int Rev Exp Pathol 1986, 28:45-78.

3. Saunders BM, Cooper AM: Restraining mycobacteria: role of granulomas in mycobacterial infections. Immunol Cell Biol 2000, 78:334-341.

4. Rojas-Espinosa O, Løvik M: Mycobacterium leprae and Mycobacterium lepraemurium infections in domestic and wild animals. Rev Sci Tech 2001, 20:219-251.

5. Ulrichs T, Kaufmann SH: New insights into the function of granulomas in human tuberculosis. J Pathol 2006, 208:261-269.

6. Clay H, Volkman HE, Ramakrishnan L: Tumor necrosis factor signaling mediates resistance to mycobacteria by inhibiting bacterial growth and macrophage death. Immunity 2008, 29:283-294.

7. Dannenberg AM Jr: Immunopathogenesis of pulmonary tuberculosis. Hosp Pract (Off Ed) 1993, 28(1):51-58.

8. Lesley R, Ramakrishnan L: Insights into early mycobacterial pathogenesis from the zebrafish. Curr Opin Microbiol 2008, 11:277-283.

9. Tobin DM, Ramakrishnan L: Comparative pathogenesis of Mycobacterium marinum and Mycobacterium tuberculosis. Cell Microbiol 2008, 10:1027-1039.

10. Davis JM, Ramakrishnan $L$ : The role of the granuloma in expansion and dissemination of early tuberculous infection. Cell 2009, 136:37-49.

11. Bouley DM, Ghori N, Mercer KL, Falkow S, Ramakrishnan L: Dynamic nature of host-pathogen interactions in Mycobacterium marinum granulomas. Infect Immun 2001, 69:7820-7831.

12. Saunders BM, Frank AA, Orme IM, Cooper AM: CD4 is required for the development of a protective granulomatous response to pulmonary tuberculosis. Cell Immunol 2002, 216:65-72.

13. Poey C, Verhaegen F, Giron J, Lavayssiere J, Fajadet P, Duparc B: High resolution chest $C T$ in tuberculosis: evolutive patterns and signs of activity. J Comput Assist Tomogr 1997, 21:601-607.

14. Tsai MC, Chakravarty S, Zhu G, Xu J, Tanaka K, Koch C, Tufariello J, Flynn J, Chan J: Characterization of the tuberculous granuloma in murine and human lungs: cellular composition and relative tissue oxygen tension. Cell Microbiol 2006, 8:218-232.

15. Puissegur MP, Botanch C, Duteyrat JL, Delsol G, Caratero C, Altare F: An in vitro dual model of mycobacterial granulomas to investigate the molecular interactions between mycobacteria and human host cells. Cell Microbiol 2004, 6:423-433.

16. Birkness KA, Guarner J, Sable SB, Tripp RA, Kellar KL, Bartlett J, Quinn FD: An in vitro model of the leukocyte interactions associated with granuloma formation in Mycobacterium tuberculosis infection. Immunol Cell Biol 2007, 5:160-168.

17. Krausgruber T, Blazek K, Smallie T, Alzabin S, Lockstone H, Sahgal N, Hussell T, Feldmann M, Udalova IA: IRF5 promotes inflammatory macrophage polarization and TH1-TH17 responses. Nat Immunol 2011, 12:231-238.

18. Satoh T, Takeuchi O, Vandenbon A, Yasuda K, Tanaka Y, Kumagai Y, Miyake T, Matsushita K, Okazaki T, Saitoh T, Honma K, Matsuyama T, Yui K, Tsujimura T, Standley DM, Nakanishi K, Nakai K, Akira S: The Jmjd3-Irf4 axis regulates M2 macrophage polarization and host responses against helminth infection. Nat Immunol 2010, 11:936-944. 
19. Benoit M, Desnues B, Mege JL: Macrophage polarization in bacterial infections. J Immunol 2008, 181:3733-3739.

20. Makino M, Baba M: A cryopreservation method of human peripheral blood mononuclear cells for efficient production of dendritic cells. Scand J Immunol 1997, 45:618-622.

21. Maeda Y, Mukai T, Spencer J, Makino M: Identification of an immunomodulating agent from Mycobacterium leprae. Infect Immun 2005, 73:2744-2750.

22. Levy L, Ji B: The mouse foot-pad technique for cultivation of Mycobacterium leprae. Lepr Rev 2006, 77:5-24.

23. McDermott-Lancaster RD, Ito T, Kohsaka K, Guelpa-Lauras CC, Grosset JH: Multiplication of Mycobacterium leprae in the nude mouse, and some applications of nude mice to experimental leprosy. Int I Lepr Other Mycobact Dis 1987, 55:889-895

24. Hashimoto K, Maeda Y, Kimura H, Suzuki K, Masuda A, Matsuoka M, Makino M: Mycobacterium leprae infection in monocyte-derived dendritic cells and its influence on antigen-presenting function. Infect Immun 2002, 70:5167-5176

25. Truman RW, Krahenbuhl JL: Viable Mycobacterium leprae as a research reagent. Int I Lepr Other Mycobact Dis 2001, 69:1-12.

26. Ridley DS, Jopling WH: Classification of leprosy according to immunity. A five-group system. Int I Lepr Other Mycobact Dis 1966, 34:255-273.

27. Hagge DA, Ray NA, Krahenbuhl JL, Adams LB: An in vitro model for the lepromatous leprosy granuloma: Fate of Mycobacterium leprae from target macrophages after interaction with normal and activated effector macrophages. J Immunol 2004, 172:7771-7779.

28. Kaplan G, Van Voorhis WC, Sarno EN, Nogueira N, Cohn ZA: The cutaneous infiltrates of leprosy. A transmission electron microscopy study. J Exp Med 1983, 158:1145-1159.

29. Postlethwaite AE, Jackson BK, Beachey EH, Kang AH: Formation of multinucleated giant cells from human monocyte precursors. Mediation by a soluble protein from antigen-and mitogen-stimulated lymphocytes. J Exp Med 1982, 155:168-178.

30. Heuts F, Gavier-Widen D, Carow B, Juarez J, Wigzell H, Rottenberg ME: CD4+ cell dependent granuloma formation in humanized mice infected with mycobacteria. PNAS 2013, 110:6482-6487.

31. Laskin DL: Macrophages and Inflammatory Mediators in Chemical Toxicity: A Battle of Forces. Chem Res Toxicol 2009, 22:1376-1385.

32. Trujillo G, O'Connor EC, Kunkel SL, Hogaboam CM: A novel mechanism for CCR4 in the regulation of macrophage activation in bleomycin-induced pulmonary fibrosis. Am J Pathol 2008, 172:1209-1221.

33. Yamamura M, Wang XH, Ohmen JD, Uyemura K, Rea TH, Bloom BR, Modlin RL: Cytokine patterns of immunologically mediated tissue damage. J Immunol 1992, 149:1470-1475.

doi:10.1186/1471-2334-13-279

Cite this article as: Wang et al: An in vitro model of Mycobacterium leprae induced granuloma formation. BMC Infectious Diseases 2013 13:279.

\section{Submit your next manuscript to BioMed Central and take full advantage of:}

- Convenient online submission

- Thorough peer review

- No space constraints or color figure charges

- Immediate publication on acceptance

- Inclusion in PubMed, CAS, Scopus and Google Scholar

- Research which is freely available for redistribution

Submit your manuscript at www.biomedcentral.com/submit
C Biomed Central 